\title{
A Facile Method Combined with Acetic Acid Modification and Electroless Plating to Fabricate Copper-plated Nylon 12 Powder for Antistatic Coating
}

\author{
ZHIPING SUN ${ }^{1,2,3}$, YUQIAO WAN ${ }^{2}$, CHUNLAN JIANG ${ }^{3}$, CHENGMEI GUI ${ }^{3,4 *}$ \\ ${ }^{1}$ School of Intelligent Manufacturing, Changzhou Vocational Institute of Engineering, Changzhou City 213164, China \\ ${ }^{2}$ School of Chemistry and Chemical Engineering, Southeast University, Nanjing City 211189, China \\ ${ }^{3}$ Jiangsu Chenguang Paint Co., Ltd. Changzhou City 213154, China \\ ${ }^{4}$ Department of Chemical and Materials Engineering, Chaohu University, Hefei City, 230601, China
}

\begin{abstract}
Metal particle could deposited on Nylon 12 (PA12) surface using electroless plating with excellent interface and distribution, but the use of noble metal as catalytic site would increase the process cost and restrict its application. In this work, we employed a facile technology combined with acetic acid etching and electroless copper plating to prepare Cu/PA12 composite powder, and it used as conductive filler for antistatic coating was also studied. Results manifested defects (hole and amorphous structure) and amide group established on etched PA12 surface, which would facilitate the destruction of the [Cu-EDTA] structure, and then the reduction of REDOX barrier. As a result, $C u$ and $\mathrm{Cu}_{2} \mathrm{O}$ particles deposited on its surface. The downward trend of volume resistivity of antistatic coating appeared the rule of slow-fast-slow. The lowest volume resistivity was about $10^{5} \mathrm{ohm} \cdot \mathrm{cm}$. This means that the dependable technology has great potential application in preparing metal/polymer composite material at a low cost.
\end{abstract}

Keywords: Electroless plating, particles, defect, powder technology

\section{Introduction}

Electroless plating is free electrochemical deposition method, which is widely used in fabricating metal/polymer composite material. The development of electroless plating has drawn extensive attention mainly because it is a simple and promising method to prepare surface-metallization material [1,2]. Metal particles, such as $A g$ [3], $\mathrm{Cu}$ [4] and $\mathrm{Ni}$ [5], in-situ plated on substrate surface with excellent interfacial characterization and dispersion, which meet all the requirements on filler of antistatic coating [6]. The key of the electroless plating reaction is the establishment of catalytic site on polymer surface, so that the plating metal ions were catalyzed reduction metal coating process of formation $[7,8]$.

It is obvious that without intermediate or cohesion in the interface between catalytic particle and substrate, the adhesion of plated coating would be greatly weakened, consequently, the modification of polymer surface is a essential technology [9]. As a result, the introduction of active group, the adsorption of catalyst and electroless plating (three-step process) are the common technology for electroless plating [10,11]. Huang et al. [9], Wang et al. [12] and Chang et al. [8] found that the modified polyethylene terephthalate surface with $-\mathrm{NH}_{2}$ and $-\mathrm{SH}$ on its surface could absorb $\mathrm{Ag}$ particles, acting as catalytic center, so that the $C u$ coatings are deposited through REDOX reaction in the presence of catalytic centers. By far the most catalytic sites, such as metal particle ( $\mathrm{Ni}$ [13], $\mathrm{Cu}$ [14], $P d$ [5] and $A g$ [9]), defect [15] and active group [16] has been reported. All of these, like $P d$ and $A g$, are noble metals, meaning that they are excellent catalytic capacity. But it has the characteristics of heavy pollution and high production cost. Non noble metal activation process has became increasingly important. Gui et al. [13] reported $\mathrm{Ni}$ particle/polymer brush was used as catalytic site on PA12 powders and $\mathrm{Cu}$-plated PA12 powders were prepared. It is speculated that the absorption mechanisms of the polymer brush for metal ion include ion exchange, physical adsorption and charge neutralization. We also found that, the surface, at the vacancy edge, has more capacity to absorb incident energy [17].

*email: guichengmei@163.com 
This effect would break the structure of metal ion ligand and release trapped metal ion in the solution, which is consistent with catalytic mechanism of noble metal in plating solution. It must be mentioned that electroless copper plating using defect as catalytic site on Nylon 12 (PA12) surface has not been studied yet. Non metal activation and simple production make it easy and low-cost to practical use.

In this work, the defect-induced electroless copper plating (two-step plated process: modification and electroless plating) was reported instead of the conventional three-step plated process (modification, activation and electroless plating) on PA12 surface. Antistatic coating used the $C u$-plated PAI2 (CCP) powder as conductive filler was prepared. The mechanisms of defect-included electroless copper plating and conductive mechanism of antistatic coating were also investigated systematically.

\section{Materials and methods}
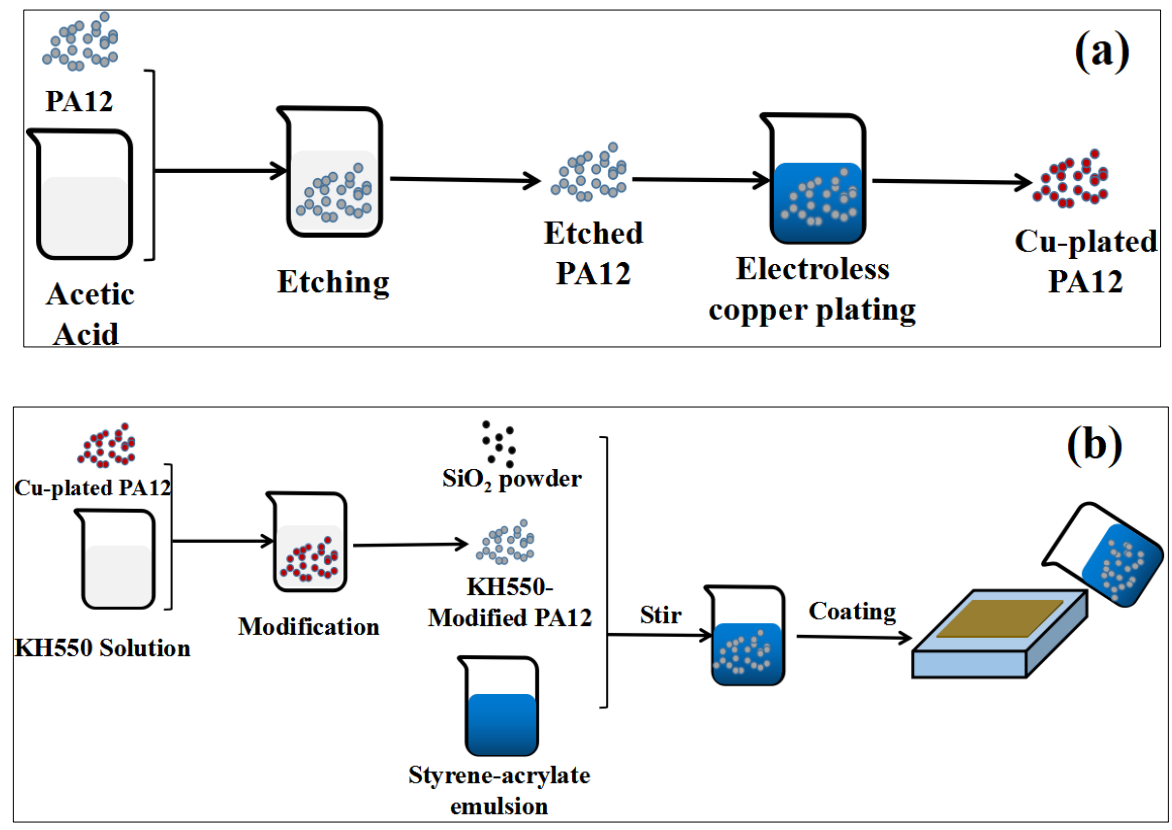

Figure 1. Schematic diagrams of electroless copper plating on PA12 powder (a) and preparation of antistatic coating (b)

\subsection{Electroless copper plating}

Figure 1a shows the schematic diagram of electroless copper plating on PA12 powder. PA12 powder was immersed in acetic acid solution at $363 \mathrm{~K}$ for $30 \mathrm{~min}$, then the $\mathrm{CH}_{3} \mathrm{COOH}$-etched PA12 powder was obtained. After that, it immersed in electroless copper plating bath at $313 \mathrm{~K}$ for $60 \mathrm{~min}$. The component of the solution is $\mathrm{CuSO}_{4}\left(15 \mathrm{~g} \cdot \mathrm{L}^{-1}\right), \mathrm{C}_{10} \mathrm{H}_{14} \mathrm{~N}_{2} \mathrm{Na}_{2} \mathrm{O}_{8}\left(\right.$ EDTA-2Na $\left.10 \mathrm{~g} \cdot \mathrm{L}^{-1}\right), \mathrm{KNaC}_{4} \mathrm{H}_{4} \mathrm{O}_{6} \cdot 4 \mathrm{H}_{2} \mathrm{O}(14 \mathrm{~g} \cdot$ $\left.\mathrm{L}^{-1}\right)$, HCHO $\left(15 \mathrm{~mL} \cdot \mathrm{L}^{-1}\right), \mathrm{C}_{10} \mathrm{H}_{8} \mathrm{~N}_{2}\left(0.02 \mathrm{~g} \cdot \mathrm{L}^{-1}\right)$ and $\mathrm{NaOH}\left(14 \mathrm{~g} \cdot \mathrm{L}^{-1}\right)$. Then, $C C P$ powder was obtained.

\subsection{Preparation of antistatic coating}

Figure $1 \mathrm{~b}$ shows the schematic diagram of the preparation of antistatic coating. The KH550 (20 g), deionized water $(8 \mathrm{~g})$ and ethanol (72 $\mathrm{g}$ ) were added in a flask equipped, and mixed at a medium speed for $30 \mathrm{~min}$, and then KH550 solution was obtained. CCP powder dropped into the system at $333 \mathrm{~K}$, and mixed at a medium speed for $60 \mathrm{~min}$. After that, the power cleaned by deionized water for 2 time, and then KH550-modified plated PA12 powders was obtained. After that, the KH550-modified powder and $\mathrm{SiO}_{2}$ particles $(0.5 \%)$ were dispersed in styrene-acrylate emulsion. The mixture was left to stir for another $60 \mathrm{~min}$, filtered the paint through 100 mesh sieve. The antistatic paint was then obtained. We put antistatic paint on glass, it was then dried to form film $(10 \mathrm{~mm} \times 10 \mathrm{~mm} \times 0.5 \mathrm{~mm})$, and then antistatic coating was obtained. The experiments' materials were purchased from Chengdu Kelong Chemical Reagent Company (China) and used without further purification. 


\subsection{Characterization}

Surface morphology and chemical composition were studied by scanning electron microscopy (SEM, JEOL, JSM-5600LV) and energy dispersive spectrometer (EDS). The phase and orientation texture of the composite particles were determined by X-ray diffraction (XRD, Rigaku D/max-2550V). The chemical structure of the samples was measured by XRD (Rigaku D/max-2550V) and X-ray photoelectron spectroscopy (XPS, Shimazu, AXIS ULTRADLD). The binding energies obtained in XPS analysis were corrected with the reference to $\mathrm{C} 1 \mathrm{~s}(284.6 \mathrm{eV})$.

\section{Results and discussions}

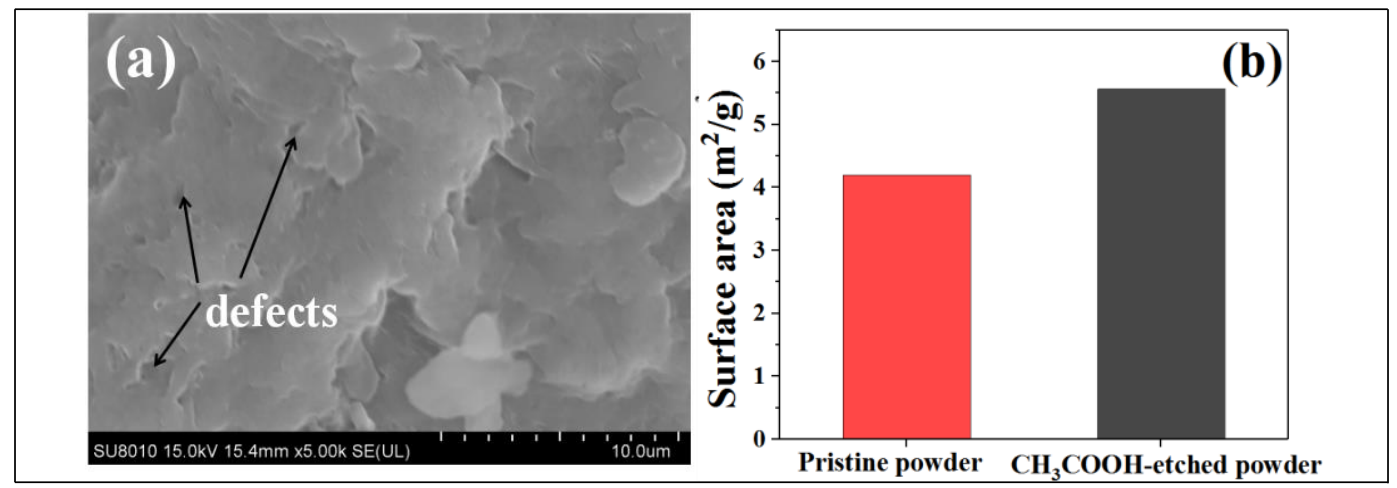

Figure 2. SEM image (a) and surface area (b) of $\mathrm{CH}_{3} \mathrm{COOH}$-etched $\mathrm{PAl} 2$ powder

As displayed in Figure 2a, there are many holes on the $\mathrm{PA} 12$ surface after $\mathrm{CH}_{3} \mathrm{COOH}$ etching. We also analysed the surface area using Brunner-Emmet-Teller method, as displayed in Figure $2 \mathrm{~b}$ and $\mathrm{S} 1$, the surface area of etched powder is $5.567 \mathrm{~m}^{2} / \mathrm{g}$, it was 33 percent higher than the one of pristine powder $\left(4.199 \mathrm{~m}^{2} / \mathrm{g}\right)$, which is consistent with the observation from SEM image. Taking the above observations into account, we conclude that the defect was established on $\mathrm{PAl} 2$ surface after $\mathrm{CH}_{3} \mathrm{COOH}$ etching.

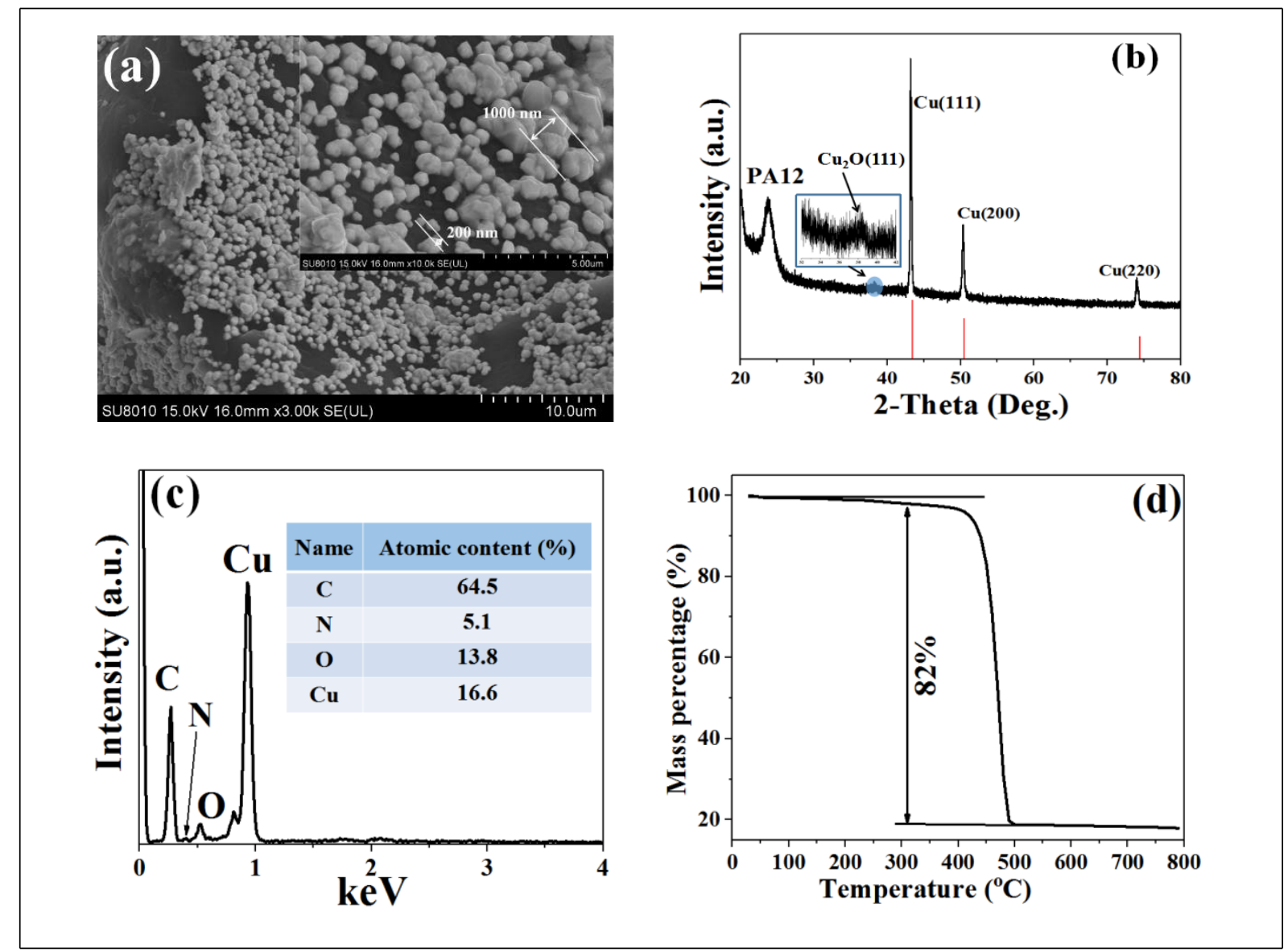



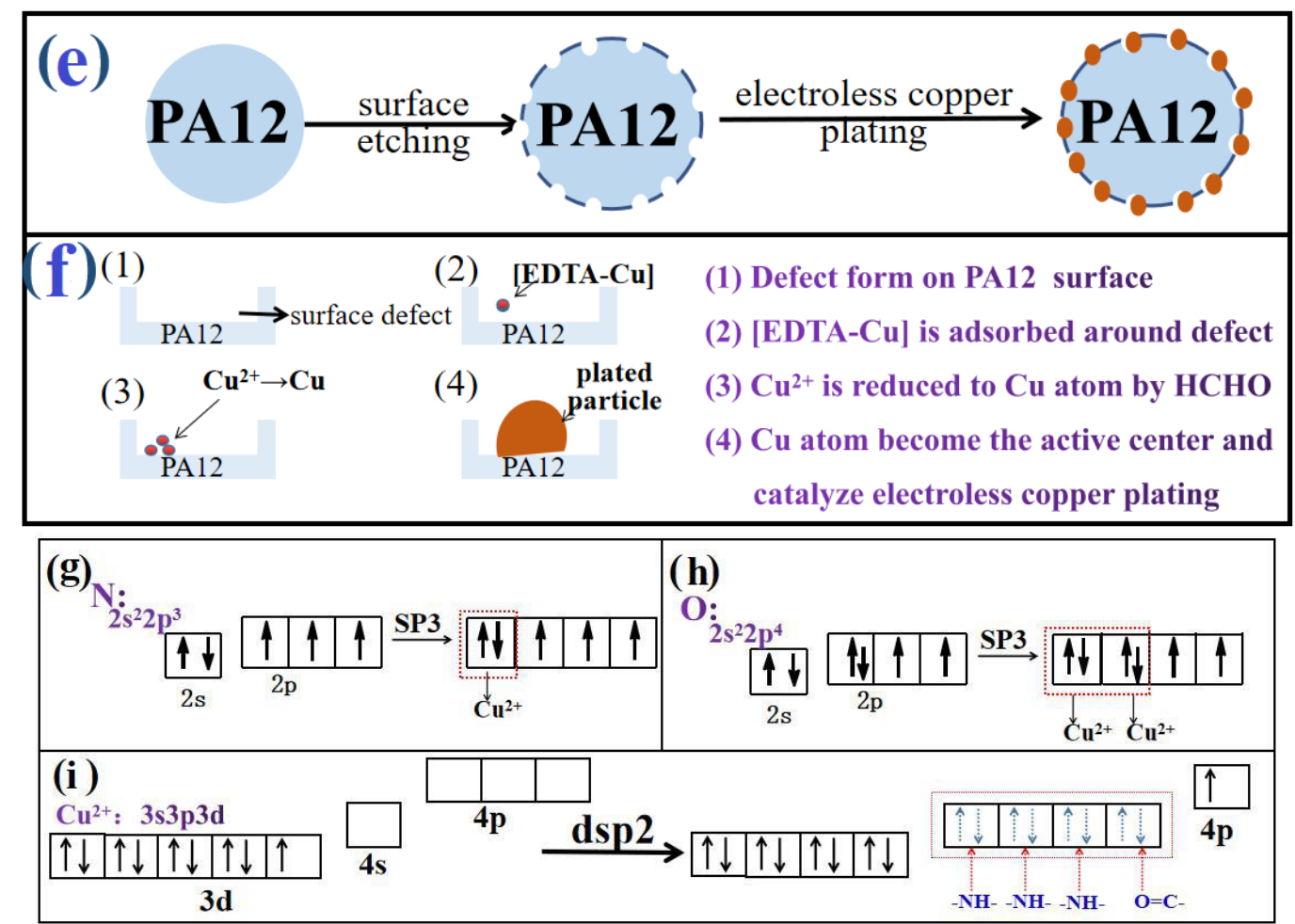

Figure 3. $S E M$ images (a), XRD (b), EDS (c) and $T G$ spectra (d) of $C C P$ powders, mechanism of defect-induced electroless copper plating (e-i)

Figure 3a represents SEM images of the CCP powder. The SEM images manifests the spherical particles deposited on $P A 12$ surface, its size are about $0.2-1 \mu \mathrm{m}$. The particles with uniformity and dense microstructure attached on $P A 12$ surface. The elements of $C u, C, N$ and $O$ are included in $E D S$ spectrum, as shown in Figure 3c. The crystal phase of $C C P$ powder was confirmed by $X R D$, as shown in Figure $3 \mathrm{~b}$. Five peaks at $24^{\circ}, 36^{\circ}, 43.3^{\circ}, 50.4^{\circ}$ and $74.5^{\circ}$ are appeared in the diffraction pattern, corresponding to $P A 12$, face-centered cubic (FCC) $\mathrm{Cu}_{2} \mathrm{O}$ (111), $\mathrm{Cu}$ (111), $\mathrm{Cu}$ (200) and $\mathrm{Cu}$ (220) characteristic peaks [9], respectively. The lattice parameters and d-spacing of $\mathrm{Cu}$ crystal are $0.361 \mathrm{~nm}$ and $0.208 \mathrm{~nm}$. Relative intensity of $\mathrm{Cu}_{2} \mathrm{O}$ (111) and $\mathrm{Cu}(111)$ characteristic peaks is the strongest in the XRD spectra, which revealed that the (111) crystal plane is preferred orientation of $\mathrm{Cu}_{2} \mathrm{O}$ and $\mathrm{Cu}$ crystal. Based on the crystallographic theory, the resulting of ratio of $F C C$ plane spacing $(d)$ is as following equation (1) $[5,18]$ :

$$
d(111): d(200): d(220)=\frac{1}{\sqrt{3}}: \frac{1}{2}: \frac{1}{2 \sqrt{2}}
$$

The (200) and (220) plane possess the more attractive force between the crystal plane due to lower $d$. Reasonably, at first, the growth rate of (200) crystal plane is the fastest, the one of (111) is the slowest. In addition, the $F C C$ plane free energy $\left(G_{l}\right)$ can be evaluated as following equation $(2)[5,18]$ :

$$
G_{1}=\frac{2 h+k}{\sqrt{h^{2}+k^{2}+l^{2}}} \times \frac{3 G_{2}}{2 V_{0}^{2}}
$$

where the $h, k$ and $l$ are Miller indices, $V_{0}$ is bond length and $G_{2}$ is bond energy. The resulting of $G_{l}$ ratio is as following equation (3) [19]:

$$
G_{1}(111): G_{1}(200): G_{1}(220)=\sqrt{3}: 2: \frac{3}{\sqrt{2}} .
$$


Based on the thermodynamical law, the particle preferably occupies the lowest energy state in a realistic crystal growth system. The $F C C \mathrm{Cu}$ and $\mathrm{Cu}_{2} \mathrm{O}$ promoted (111) growth and inhibited the other crystal orientation growth. As a result, preferred orientation of (111) crystal plane tends to be stronger gradually with the growth of grain. $T G$ test was employed to evaluate $C u$-deposited quantity on the $C P P$ surface $\left(M_{\text {plated particle }} M_{(P A 12+\text { platedparticle })}\right)$. Figure 3d represents $T G$ spectrum of $C P P$ powder. As shown in Figure $3 \mathrm{~d}$, the $M_{\text {plated particle }} / M_{(P A 12+\text { platedparticle })}$ is $18 \%$, which is almost $72 \%$ of the one of $C C P$ by $N i$ catalyzed electroless plating [13]. But it is more cost competitiveness compared with the reported work due to non metal activation and simple production.

Overall, a facile technology combined with etched modification and electroless copper plating to preparate $C u / P A 12$ composite powder was achieved. As shown in Figure $3 \mathrm{e}$ and $\mathrm{f}$, it can be concluded that there are four important processes: First, defect form on $\mathrm{PA} 12$ surface after $\mathrm{CH}_{3} \mathrm{COOH}$ etching. Second, [EDTA- $\mathrm{Cu}$ ] ligands is adsorbed around defect due to its high surface energy. Third, [Cu-EDTA] ligands stability is reduced and $C u^{2+}$ is reduced to $\mathrm{Cu}$ atom by $\mathrm{HCHO}$. At last, $\mathrm{Cu}$ atoms deposited on $P A 12$ surface $[13,20]$. The key point is the intermediate step. The plating bath can be stable because of the formation of EDTA-Cu (ligand), and the REDOX barrier is enhanced, as a result, the $C u^{2+}$ cannot be reduce by the reductant $(\mathrm{HCHO})$. The reduction of [Cu-EDTA] stability would facilitate the electroless plating. The reduction process could be expressed as the following equation (4) and (5) $[13,20]$.

$$
\begin{gathered}
\mathrm{Cu}^{2+}+2 \mathrm{HCHO}+4 \mathrm{OH}^{-} \longrightarrow \mathrm{Cu} \downarrow+2 \mathrm{HCOO}^{-}+2 \mathrm{H}_{2} \mathrm{O}+\mathrm{H}_{2} \uparrow \\
2 \mathrm{Cu}^{2+}+\mathrm{HCHO}+5 \mathrm{OH}^{-} \rightarrow \mathrm{Cu}_{2} \mathrm{O} \downarrow+\mathrm{HCOO}^{-}+3 \mathrm{H}_{2} \mathrm{O}
\end{gathered}
$$

Polar solvent molecule $\left(\mathrm{CH}_{3} \mathrm{COOH}\right)$ and amide group (-NHCO-) are tending to form excellent binding force (hydrogen bonding and polar bonds), and then break the binding force (intermolecalar force and hydrogen bonding) during polymer chains. The chains gradually dissociated form PA12 surface. This process is firstly breaking bond between the chain, then the reduction of its order, swelling at last. It can reduce the $P A 12$ crystallinity from the outer to the center region. The surface energy and $N H C O$ - content would enhanced after etching.

In addition, as shown in Figure $3 \mathrm{~g}, \mathrm{~h}$ and $\mathrm{i}$, lone pair electrons form both $N(2 s 22 p 3)$ and $O(2 s 2$ 2p4) SP3 hybrid orbitals could occupied the $\mathrm{Cu}^{2+} d s p 2$ hybrid orbital. As a result, a strong force can be establish between amide group and $[C u$-EDTA], the force itself naturally takes on the form of coordinated bonds $(\mathrm{Cu}-\mathrm{N}$ and $\mathrm{Cu}-\mathrm{O})[9,12]$. The $[\mathrm{Cu}-\mathrm{EDTA}]$ stability was reduced by the coordination effect, its structure was break and $C u^{2+}$ was released [13], as displayed in Figure 3f, $\mathrm{g}$ and $\mathrm{h}$. On the other hand, the swelling and dissolution would facilitate the formation of hole on the PA12 surface. The surface defect (amorphous structure and hole) and amide group of their high capillary imbibition and chemical activity also would reduce the $\left[C u\right.$-EDTA] stability [21], which would release trapped $C u^{2+}$. The free $\mathrm{Cu}^{2+}$ was reduced by $\mathrm{HCHO}$ directly, as shown in Figure $3 \mathrm{f}$.

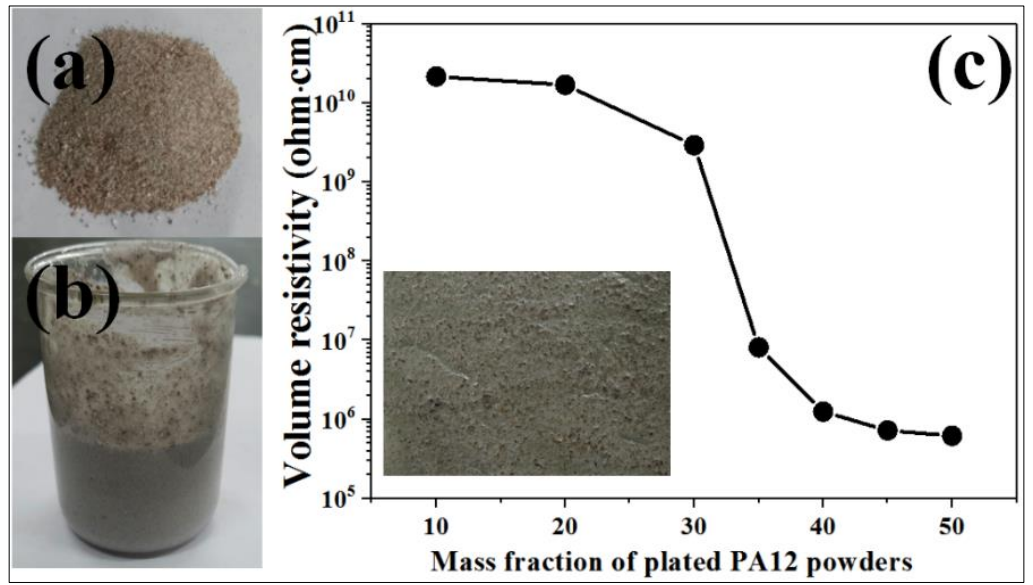




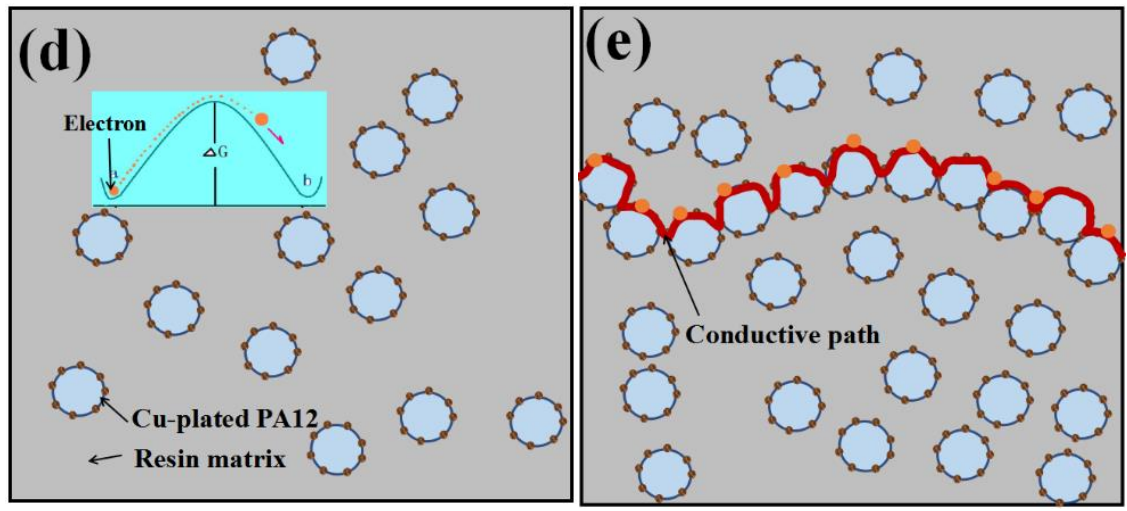

Figure 4. Photographic pictures of the $C C P$ powder (a) and Antistatic paint (b), volume resistivity (c) and conductive mechanism (d) (e) of antistatic coating

An antistatic paint (Figure 4b) was fabricated with styrene-acrylate resin as bonding agent and $C C P$ powder as conductive filler. Figure $4 \mathrm{c}$ manifests volume resistivity of antistatic coating with different mass fraction of $C C P$ powder. The test method is displayed in Figure S2. It was observed that the volume resistivity of antistatic coating with $10 \%, 20 \%, 30 \%, 35 \%, 40 \%, 45 \%$ and $50 \% C C P$ powder are $2.1 \times 10^{10}$ $\mathrm{ohm} \cdot \mathrm{cm}, 1.6 \times 10^{10} \mathrm{ohm} \cdot \mathrm{cm}, 2.9 \times 10^{9} \mathrm{ohm} \cdot \mathrm{cm}, 8.1 \times 10^{6} \mathrm{ohm} \cdot \mathrm{cm}, 1.2 \times 10^{6} \mathrm{ohm} \cdot \mathrm{cm}, 7.3 \times 10^{5} \mathrm{ohm} \cdot \mathrm{cm}$ and $6.1 \times 10^{5} \mathrm{ohm} \cdot \mathrm{cm}$, respectively. Obviously, the downward trend of volume resistivity of antistatic coating appeared slow firstly and fast afterwards, at last $S$-curve. In addition, a considerable reduction of volume resistivity appears in the pattern (30-40\% CCP), which is the so-called percolation threshold. The $C C P$ powder is "geometrically isolated"' at low filler content (Figure 4d) [22]. The conducting mechanism is the quantum tunnel effect of conductive particles resulting in the higher electronic transition barrier $(\Delta G)$ and volume resistivity. The distance between neighboring plated powder would reduce with the increase of filler density, and then percolation channel was established when the content of filler up to a certain extent. (Figure 4e), the carrier can transport in the continuous plated $P A 12$ network [23,24]. The $\Delta G$ is reduced significantly, therefore, volume resistivity is quickly reduced.

\section{Conclusions}

A facile technology combined with etched modification and electroless copper plating to fabricate $C C P$ powder is successfully established. The hole, amide group and amorphous structure formed on $\mathrm{CH}_{3} \mathrm{COOH}$-etched $\mathrm{PA} 12$ surface, which can break the structure of [Cu-EDTA] ligand, subsequently, the $\mathrm{REDOX}$ barrier of electroless copper plating was reduced. As a result, $\mathrm{Cu}$ and $\mathrm{Cu}_{2} \mathrm{O}$ spherical particles with a diameter of about $0.2-1 \mu \mathrm{m}$ deposited on PA12 surface. The downward trend of volume resistivity of antistatic coating appeared the rule of slow-fast-slow. The lowest volume resistivity was approximately $10^{5} \mathrm{ohm} \cdot \mathrm{cm}$.

This dependable technology has great potential application in preparing metal-polymer powder for additive manufacturing, conductive coating and electromagnetic shielding.

Acknowledgements. The authors would like to thank Natural Science Foundation of the Jiangsu Higher Education Institutions of China (Grant No. 18KJB430006) and Startup Foundation for Advanced Talents of the Changzhou Vocational Institute of Engineering (No. 11130900118003).

\section{References}

1. X.H. WU, G. HONG, X.T. ZHANG., Electroless Plating of Graphene Aerogel Fibers for Electrothermal and Electromagnetic Applications. Langmuir 35 (2019) 3814-3821. 
2. Y.F. WANG, Y. HONG, G.Y. ZHOU, W. HE, Y. WANG., Compatible $\mathrm{Ag}^{+}$Complex-Assisted Ultrafine Copper Pattern Deposition on Poly(ethylene terephtalate) Film with Micro Inkjet Printing. ACS Appl. Mater. Interfaces 11 (2019) 44811-44819.

3. R.F. ZUO, J.Y. CHEN, Z.H. HAN, et al. Electroless silver plating on modified fly ash particle surface. Appl. Surf. Sci. 513 (2020) 145857.

4.J.J. HUANG, L.L. XU, D.F. ZHAO, et al. A facile process to fabricate metal coating on PET sheet: Preparation of highly active polymer brush/Ag particle and Its application in electroless copper plating. Chem. Eng. J. 383 (2020) 123199.

5. J.J. HUANG, C.M. CHEN, H.D. MA, et al. Surface metallization of PET sheet: Fabrication of Pd nanoparticle/polymer brush to catalyze electroless nickel plating. Compos. Sci. Technol. 202 (2021) 108547.

6. X. LIU, D.K. ZHANG, Z.G. GUO. A Facile Modifier-free Approach to Fabricate Antistatic Superhydrophobic Composite Coatings with Remarkable Thermal Stability and Corrosion Resistance. J Bionic Eng. 17 (2020) 421-435.

7. T. BOETTCHER, S. SCHAEFER, M. ANTONI, et al. Shape-Selective Electroless Plating within Expanding Template Pores: Etching-Assisted Deposition of Spiky Nickel Nanotube Networks. Langmuir 35 (2019) 4246-4253.

8. Y. CHANG, Y. TAO, Q. ZHANG, et al. Selective adsorption of catalyst and copper plating for additive fabrication of conductive patterns and through-holes. Electrochim Acta 158 (2015) 7-12.

9. J.J. HUANG, Y.ZHANG, M.M. YUAN, et al. A facile process to fabricate electroless plating on PET sheet: Effects of surface roughness on adhesive force, electronic and structural properties of copper coating. J. Taiwan Inst. Chem. 97 (2019) 406-413.

10. T. ZHANG, X.L. WANG, T.J. LI, C. GUO. Fabrication of flexible copper-based electronics with high-resolution and high-conductivity on paper via inkjet printing. J. Mater. Chem. C 2 (2014) 286-294. 11. J.J. HUANG, C.A. TIAN, J. WANG, et al. Fabrication of selective electroless copper plating on PET sheet: Effect of PET surface structure on resolution and adhesion of copper coating. Appl. Surf. Sci. 458 (2018) 734-742.

12. Y. WANG, Y. WANG, J.J. CHEN, et al, A facile process combined with inkjet printing, surface modification and electroless deposition to fabricate adhesion-enhanced copper patterns on flexible polymer substrates for functional flexible electronics. Electrochimica Acta 218 (2016) 24-31.

13. C.M. GUI, C.G. YAO, J.J. HUANG, et al, Preparation of polymer brush/Ni particle and its application in electroless copper plating on PA12 powder. Appl. Surf. Sci. 506 (2020) 144935.

14. W. XU, M. ZHUANG, Z. CHENG, Environmentally friendly copper metallization of ABS by $\mathrm{Cu}-$ catalysed electroless process. Rare Metal Mat. Eng. 45 (2016) 1709-1713.

15. C.M. GUI, C.Z. CHEN, J.J. HUANG, et al, Preparation of nickel/PA12 composite particles by defectinduced electroless plating for use in SLS processing. Sci. Rep. 8 (2018) 13407.

16. Q.H. LI, G.C. QIN, J.J. HUANG, et al. POLYMER BRUSH-INDUCED ELECTROLESS COPPER PLATING TO FABRICATE Cu/CaCO ${ }_{3}$ COMPOSITE POWDER. Surf. Rev. Let. 27 (2021) 2050053.

17. F.J. KNORR, D. ZHANG, J.L. MCHALE, Influence of $\mathrm{TiCl}_{4}$ treatment on surface defect photoluminescence in pure and mixed-phase nanocrystalline $\mathrm{TiO}_{2}$. Langmuir 23 (2007) 8686.

18. Z.P. SUN, J.J. HUANG, Q. LIU, M. GAO, M.Y. LI, F. ZHAO, W. CHENG, B. TANG, Effects of temperature on Ni coating on poly(ethylene terephthalate) substrate modified with primer. J Mater Sci: Mater Electron 27(6) (2016) 5892-5898.

19. W.H. LUO, W.Y. HU, K.L. SU, F.S. LIU, The calculation of surface free energy based on embedded atom method for solid nickel. Appl. Surf. Sci. 265 (2013) 375-378.

20. D.X. CHEN, Y. ZHANG, T.S. BESSHO, et al, Layer by layer electroless deposition: An efficient route for preparing adhesion-enhanced metallic coatings on plastic surfaces. Chem. Eng. J. 303 (2016) 100-108.

21. Z.N. SHU, X. WANG, Environment-friendly Pd free surface activation technics for ABS surface. Appl. Surf. Sci. 258 (2012) 5328-5331. 
22. M. KUNIMOTO, A. OTOMO, N. TAKAHASHI, et al. Theoretical analysis of the influence of surface defects on the reactivity of hypophosphite ions. Electrochim. Acta 113 (2013) 785-791.

23. I.V. ANTONOVA. M. GULYAEV, E. SAVIR, et al, Charge storage, photoluminescence, and cluster statistics in ensembles of Si quantum dots. Phys. Rev. B 77 (2008) 125318.

24. I. BALBERG, Electrical transport mechanisms in three dimensional ensembles of silicon quantum dots. J. Appl. Phys. 110 (2011) 061301

Manuscript received: 27.02 .2021 